\title{
Risk Management in Process Industry - Practical Approach in Poland
}

\author{
ANDRZEJ KOZAK \\ Office of Technical Inspection \\ 02-353 Warsaw \\ 34, Szczesliwicka Street, Poland
}

\begin{abstract}
As one of the risk analysis methods HAZOP has been successively and commonly applied for many years since it enables the future user of a process installation to check the project in terms of security in a way that minimises the costs of changes. This paper refers to the weak points of risk management procedures in the process industry which may occur when applying analysis techniques during the plant life cycle and typical common errors made during the HAZOP analysis. A technical assessment of the industrial environment and installations is proposed as a background to RBI procedures.
\end{abstract}

Key words: Risk-based Inspection [RBI], Hazard and Operability Study [HAZOP], life cycle cost [LCC].

\section{Introduction}

The Office of Technical Inspection (Polish: UDT) performs about half a million inspections of pressure equipment throughout the country per year.

The recorded number of failures of this equipment has been relatively low for many years - about 10 cases of technical failures per year. The purpose of this study is not to demonstrate the advantages of the various techniques of risk analysis, but to give guidance regarding the most common weak points of various techniques and the most common difficulties associated with risk management in the installation life cycle. It should also be clear that without proper actions in the earlier phases, performance of RBI type inspections during the operational phase is seriously impeded.

\section{Safety management in plant life cycle}

It is possible to take a risk and formulate a premise that approximately $75 \%$ of incidents and failures result from the lack or insufficiency of the safety culture in a specific plant in the process industry (HSE data [1] relating to the gas industry). At the same time, the most favourable case is that the plant risk management is implemented over the entire life cycle e.g. "from the cradle to the grave" also life cycle cost [2].

A comprehensive approach that the Office of Technical Inspection has successfully applied for several years in Poland is presented in Fig. 1. The initial phase, or the Development Application Stage, should end with the execution and implementation of the proposals arising from the preliminary risk analysis. During the next stage, the Design Stage which is a comprehensive, both qualitative and quantitative, risk analysis should be performed. Also an explosion protection plan, an action plan in case of failure, and a crisis management plan should be prepared. The next phase, the Construction and Commissioning Stage, is intended to supervise the execution and practical implementation of the conclusions and recommendations from the previous stages. The last phase, the Operational Stage, is the time for "reaping the fruits" which have previously been worked out, including the Risk-based Inspection [3] [4]

\subsection{Development Application Stage.}

Technique: PHA or preliminary HAZOP and C-HAZOP. Weak points:

Errors occurring in this phase are most often errors committed by a licensor or a contractor preparing the technical documentation based on the purchased license. Thus, they are the errors "purchased" by the future operator of the plant:

a. lack of the maturity of technology and/or technical documentation - technology errors on the part of a licensor.

It often happens that a technology license is bought, in which the kinetics of the processes are not fully known or verified experimentally. Other cases include gaps in the technical documentation such as inadequately known and not thoroughly studied corrosion processes. Or, the process apparatus is not well-chosen or incorrectly calculated, neglecting a variety of parallel or side processes. 


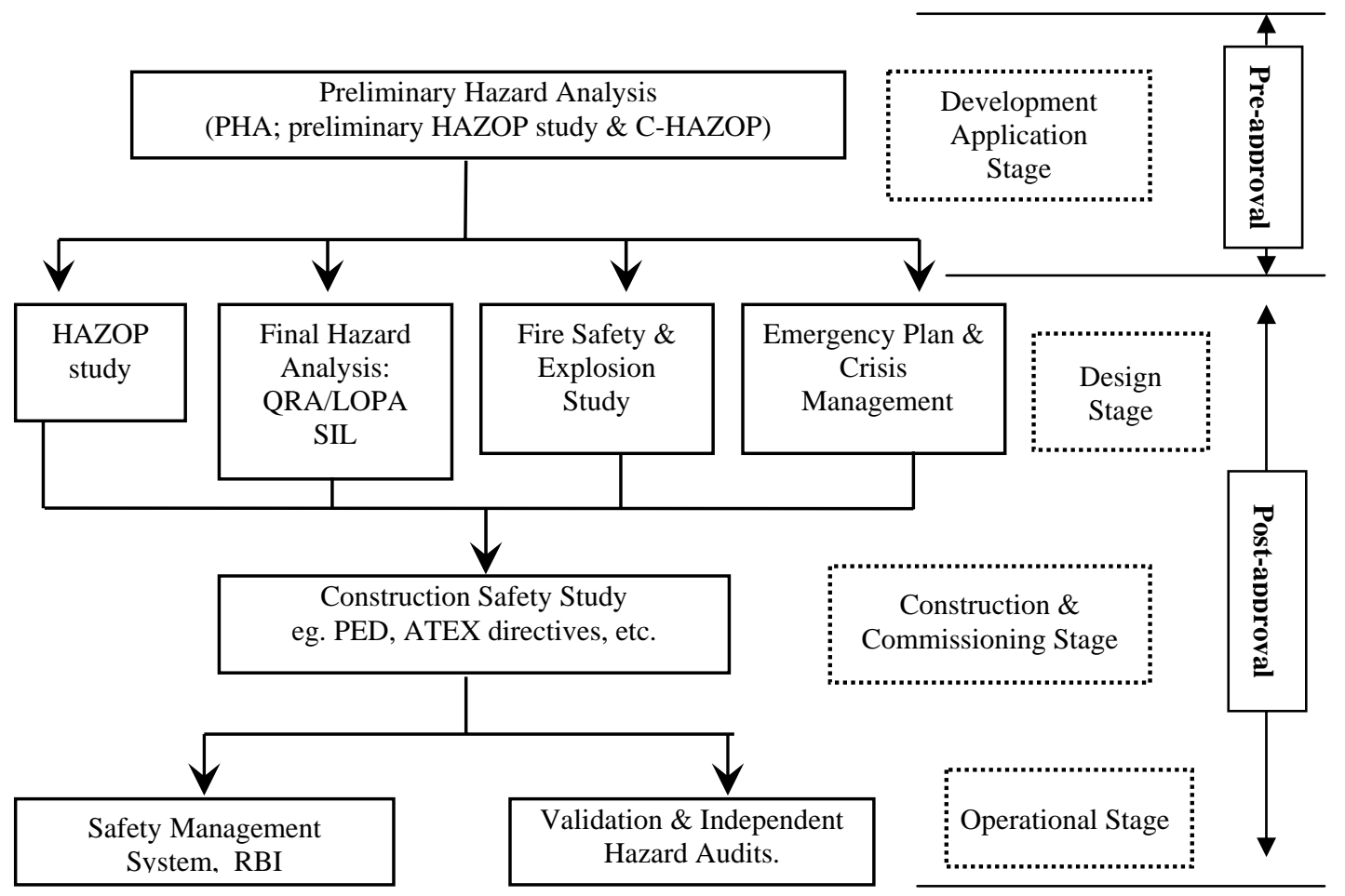

Fig. 1 Risk management chart within the entire plant life cycle in the process industry

b. lack of the implementation of a contracted technical specification - errors of the so-called basic documentation on the part of a licensor or contractor. In the majority of cases, this means omissions or errors in the application of technical standards and best engineering practices. It is a frequent case that appears or is not noticed in due time by the so-called young and dynamic contractor team.

\subsection{Design Stage - common errors.}

The most serious mistakes during this phase can be made by the owner of the plant. Mistakes made by other parties involved in the investment project result rather from the owner's tacit acquiescence.

Technique: HAZOP

Weak points:

1. missing plant and/or corporation risk matrix,

2. lack of interest of the end-user's management staff,

3. formal qualitative analysis only,

4. the plant manager is not interested in the analysis or does not have enough knowledge or experience,

5. formal analysis only - very big binder/paper file but without conclusions or important data.

The first and very important stage is the design stage. As concerns plant safety, a well executed design stage is like the foundations for a building. Errors made at this stage are usually irreparable at the following stages. The plant management staff must clearly declare which losses are acceptable, tolerable- acceptable or unacceptable. If it is not the case, the UDT inspectors will use UDT- universal risk matrix.

\subsubsection{Case study from HAZOP analysis}

In the course of HAZOP analysis, it is crucial to determine which of the detected hazards may be contained:

a. individually by means of actions undertaken by the operator

b. by departmental chemical rescue teams and which of the detected events should be subject to further analysis, e.g. Layer of Protection Analysis (LOPA), Quantitative Risk Analysis (QRA), Safety Integrity Level (SIL) analysis, etc. Fig. 2 shows real-life data obtained during HAZOP analysis of the process installation in the petrochemical industry conducted by the author. The installation was divided into 6 analytical nodes.

Attention should be focused on Node 5 in which there were 8 hazardous events identified and Node 1 in which there were 5 hazardous events identified that required 
the intervention of the installation operator - Fig. 2. Hazardous events (that simultaneously occurred in Nodes 1 and 5) may transform into a top event that leads to a catastrophic situation. It should be emphasized that, the number of correct human actions undertaken under stress is limited. The detection and prevention of such hazards depends on the experience of the HAZOP team members.

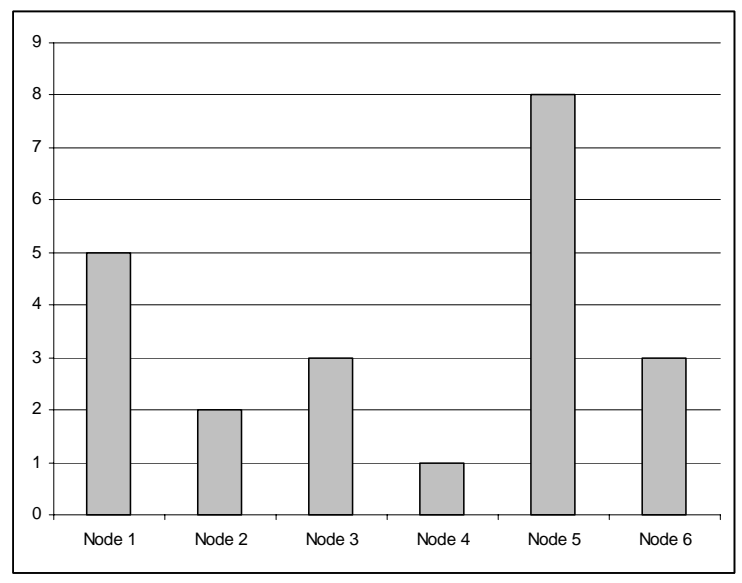

Fig. 2 Number of interventions undertaken by operations in response to hazardous events detected during HAZOP analysis for individual nodes.

\subsection{Common errors - Construction and Commissioning Stage.}

At this stage, the major errors are primarily committed by the plant owner together with its operator.

The most frequent errors include:

1. lack of experience with conformity assessment procedures,

2. not certified staff and service for emergency shut down or lock systems,

3. device not reliable enough to be applied in control or emergency shout-down (ESD) loops.

The design itself is usually made by an experienced and certified team but the construction is carried out by an enterprise preparing a tender under the pressure of cost cutting procedures. Cost cutting is usually in conflict with safety! According to [2], the life cycle cost analysis made by third party experts could be counteractive.

\subsection{Common errors - Operational Stage}

Small improvements without a thorough analysis, particularly of the flow dynamics and the possibility of preventing an ignition reaction.

\subsubsection{Case study from an organic industry in Poland:}

After the commissioning that followed a minor upgrade, the reactor piping was broken - Fig. 3. Why? What was the matter? A piston pump was replaced with a rotary pump. The rotary pump mixed liquids in a more turbulent way than the piston one and injected an additional amount of energy into the system which was sufficient to start a chemical reaction in the piping instead of the reactor - 1-2m ahead of the reactor resistant to corrosion.

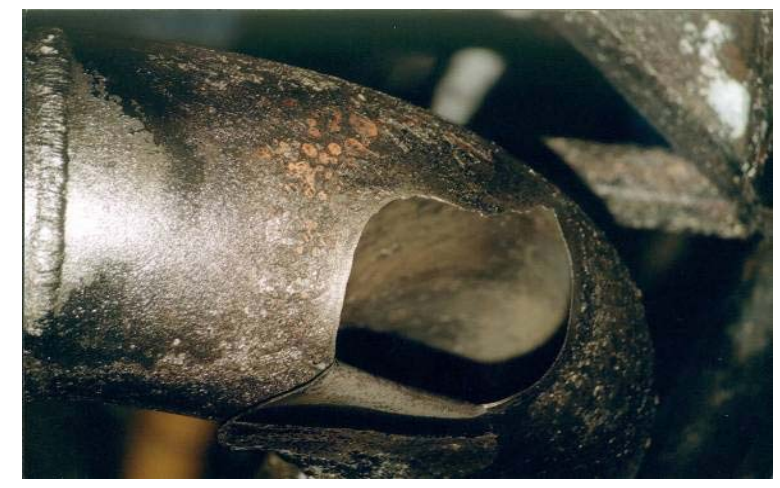

Fig. 3 A DN250mm tube broken by pressure a few weeks after the minor plant modernization.

\subsection{Too many different alarms and signals in the control room}

Usually, a well-educated and experienced engineer can effectively identify and analyse a maximum of 5 different signals per minute during 8 hours of work. If there are more signals, the control room engineer can get tired and unfocused. And then the chance of making a mistake increases rapidly.

\section{Risk-Based Inspection}

The RBI method is a modern inspection method primarily applicable in the case of a large difference between the inspection time and the time required to prepare the plant for testing by traditional methods such as the water-pressure test and the plant start-up time after the inspection testing.

A flow chart of an RBI process is shown in Fig. 4.

3.1 A case study based on a practical inspection time value:

Customary inspection in a high-tech catalyst reactor plant in the petrochemical industry:

1. inspection time - 16 hours 
2. preparation time for inspection - 3 days (72 hours)

3. plant start-up time after inspection -4 days (96 hours)

Generally, customary inspection staff is not aware of what happened at the previous stages.

Risk-based inspection time is introduced in this plant without a production break. To perform an inspection using this method, it is necessary to prepare documents at previous stages of the plant life cycle [5].

The application of RBI requires long-time cooperation with different specialists, especially:
a. maintenance staff,
b. plant corrosion specialist,
c. plant engineers,
d. others (if needed),

and is much better and cheaper when this cooperation and data collection start at the initial stage of the plant life time. Additional benefits for the plant operator: the plant is ready to implement the Reliability-based Inspection (RBI)

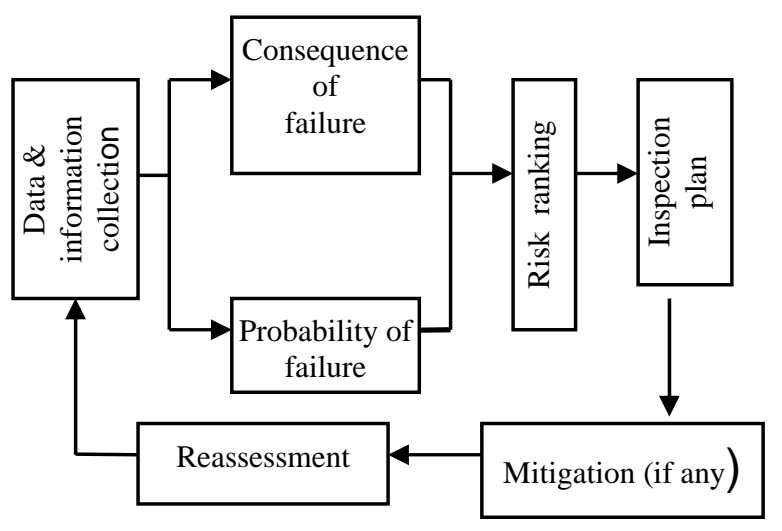

Fig. 4 Risk-based Inspection flow chart (acc. to [4]).

\section{Conclusions}

The plant safety system management should be carried out by a multi-disciplinary team of specialists with relevant knowledge and experience of the technology and plant. Errors made in the earlier phases have a tendency to accumulate and multiply losses during the following stages. In the course of a complex risk analysis, it is essential to take into consideration the possible mistakes that are likely to happen. In order to avoid them, a group of competent specialists should be formed as a working team, without economic interests to reduce expenditures on safety. The most dangerous mistakes often made by the team are hidden mistakes, especially those that remain undetected at different stages of safety analysis. If not accompanied by a deep insight into the technology of the process, even the best formal approach may lead to shortcomings in the analysis. Potential sources of mistakes, especially those referred to as an example in this paper, shall be taken into account during a comprehensive analysis. A simple mistake made at an early stage can cost the end user a lot of money when trying to correct it during the operational phase, possibly after the first accident.

\section{References:}

[1] Health and Safety Executive Report - CRR No. 139

[2] Standard EN - 60300-3-3 "Dependability management - Part 3-3. Application guide -Life cycle costing.

[3] Standard API RP No. 581 "Risk-based Inspection. Base Resource Document”.

[4] Standard API No. 580 "Risk-based Inspection. Recommended Practice".

[5] Andrzej Kozak "Reliability Analysis and RBI Planning for Industrial Vessels". International Applied Reliability Symposium. Amsterdam 2011. Symposium Proceedings.

\section{Bibliographical note:}

Dr Andrzej Kozak is the manager of the Department of Functional and Process Safety at the Office of Technical Inspection (UDT) in Warsaw, Poland and a lecturer at the Technical University of Łódź. He graduated from the Cracow University of Technology, where he received a Master's Degree in Chemical and Process Engineering. He holds a Ph.D. from the Polish Academy of Sciences. He has 35 years experience in process industry and safety management. He has received the title of a Certified Reliability Professional and he is a member of the American Chemical Society 